\title{
The first-in-class anti-EGFR antibody mixture Sym004 overcomes cetuximab-resistance mediated by EGFR extracellular domain mutations in colorectal cancer
}

Francisco Javier Sánchez-Martín ${ }^{1}$, Beatriz Bellosillo ${ }^{1,2}$, Mariona Gelabert ${ }^{1}$, Alba Dalmases $^{1,2}$, Israel Cañadas ${ }^{1}$, Joana Vidal ${ }^{3}$, Alejandro Martinez ${ }^{1,3}$, Guillem Argilés ${ }^{4}$, Giulia Siravegna ${ }^{5,6}$, Sabrina Arena ${ }^{5,6}$, Klaus Koefoed ${ }^{8}$, Laura Visa ${ }^{3}$, Oriol Arpí ${ }^{1}$, Ivan D Horak $^{8}$, Mar Iglesias ${ }^{2}$, Christopher Stroh ${ }^{7}$, Michael Kragh ${ }^{8}$, Ana Rovira ${ }^{1}$, Joan Albanell $^{1,3,9}$, Josep Tabernero ${ }^{4}$, Alberto Bardelli ${ }^{5,6}$, Clara Montagut ${ }^{1,3}$

${ }^{1}$ Cancer Research Program, IMIM, Hospital del Mar, Barcelona, Spain; ${ }^{2}$ Pathology Department, Hospital del Mar, Barcelona, Spain; ${ }^{3}$ Medical Oncology Department, Hospital del Mar, Barcelona, Spain; ${ }^{4}$ Medical Oncology Department, Vall d’Hebron Institute of Oncology (VHIO), Barcelona, Spain; ${ }^{5}$ Candiolo Cancer Institute - FPO, IRCCS, Candiolo, Torino, Italy; ${ }^{6}$ University of Torino, Department of Oncology, SP 142, KM 3.95, 10060 Candiolo, Torino, Italy; ${ }^{7}$ Merck-Serono; ${ }^{8}$ Symphogen A/S, 2750 Ballerup, Denmark ${ }^{9}$ Universitat Pompeu Fabra, Barcelona, Spain

Running title: Sym004 overcomes EGFR mediated cetuximab resistance

Keywords: Colorectal neoplasms, EGFR, Sym004, resistance, cetuximab

Financial support: This work was supported by PI12/00989, PI15/00457, DTS15/00048 and by the Xarxa de Banc de Tumors de Catalunya. 


\section{Correspondence to:}

Clara Montagut, Medical Oncology Department, Hospital del Mar, Passeig Maritim 25-

29, Barcelona 08003, Spain. Telephone: + 3493248 3137; e-mail address: cmontagut@hospitaldelmar.cat

Potential Conflicts of Interest: None to Disclose

Word count: 3831

Total number of figures: 4

Total number of tables: 2 


\section{Statement of translational relevance}

The anti-EGFR monoclonal antibody cetuximab is effective for the treatment of patients with KRAS and NRAS wild-type metastatic colorectal cancer (mCRC). Unfortunately, patients ultimately develop disease progression, driven by acquisition of mutations in the extracellular domain of EGFR in approximately $20 \%$ of the cases. Therapeutic strategies to circumvent resistance driven by ECD mutations are an unmet need. In the present study we evaluate the efficacy of Sym004 in in vitro and in vivo cetuximab resistant CRC models and find that Sym004 is a valid strategy to treat CRC tumors harboring EGFR ECD mutations. As a proof-of-concept, one patient with an EGFR ECD mutation (G465R) benefited from treatment with Sym004. Accordingly, identification of EGR ECD mutations in cetuximab refractory $\mathrm{mCRC}$ patients will be paramount to design additional lines of therapy that include Sym004. 


\begin{abstract}
Purpose: Approved anti-EGFR antibodies cetuximab and panitumumab provide significant clinical benefit in patients with metastatic colorectal cancer (MCRC). However, patients ultimately develop disease progression, often driven by acquisition of mutations in the extracellular domain (ECD) of EGFR. Sym004 is a novel 1:1 mixture of two non-overlapping anti-EGFR monoclonal antibodies that recently showed promising clinical activity in a phase I trial in MCRC. Our aim was to determine the efficacy of Sym004 to circumvent cetuximab resistance driven by EGFR ECD mutations.
\end{abstract}

Experimental Design: Functional studies were performed to assess drug-receptor binding as well as ligand-dependent activation of individual EGFR mutants in the presence of cetuximab, panitumumab and Sym004. Cell viability and molecular effects of the drugs were assayed in cetuximab-resistant cell lines and in tumor xenograft models. Efficacy of Sym004 was evaluated in patients progressing to cetuximab that harbored EGFR mutation in the post-cetuximab tumor sample.

Results: Contrary to cetuximab and panitumumab, Sym004 effectively bound and abrogated ligand-induced phosphorylation of all individual EGFR mutants. Cells resistant to cetuximab harboring mutations in EGFR maintained sensitivity to Sym004, which was consistent with an effective suppression of EGFR downstream signaling, translating into profound and sustained tumor regression in the xenograft model. As proof of principle, a patient with a tumor harboring an EGFR mutation (G465R) following cetuximab therapy benefited from Sym004 therapy.

Conclusions: Sym004 is an active drug in MCRC resistant to cetuximab/panitumumab mediated by EGFR mutations. EGFR mutations are potential biomarkers of response to Sym004 to be evaluated in ongoing large clinical trials. 


\section{Introduction}

Approved monoclonal antibodies $(\mathrm{mAb})$ directed against the epidermal growth factor receptor (EGFR) --cetuximab and panitumumab-- provide significant survival benefit to patients with wild-type KRAS and NRAS metastatic colorectal cancer (MCRC) and are now standard components of treatment regimens for these patients, either alone or in combination with chemotherapy $(1,2)$. Unfortunately, clinical efficacy of anti-EGFR $\mathrm{mAb}$ is limited by the emergence of acquired resistance that eventually develops in all initially responding tumors.

Preclinical models, tumor tissue genotyping, and circulating tumor (ct) DNA obtained from patients following progression to cetuximab or panitumumab have led to the identification of several mechanisms that mediate acquired resistance to anti-EGFR therapy in MCRC (3-5). The most frequent strategy used by cancer cells to evade EGFR blockade involves downstream pathway reactivation by either mutations or, less frequently, amplification in EGFR downstream effectors including KRAS, NRAS or activation of alternative tyrosine kinase receptors such as HER2 or c-MET (6-8). A second mechanism of resistance entails mutations in the extracellular domain (ECD) of EGFR that prevent binding of the drug to the receptor. The first reported mutation of resistance was a change of a Serine to an Arginine at position 492 (S492R) (3), which occurs in approximately $16 \%$ of patients treated with cetuximab, as assessed by ctDNA $(9,10)$. More recently, other mutations in EGFR ECD have been identified in patients treated with anti-EGFR mAb (R451C, K467T, G465R) and in preclinical models of resistance to anti-EGFR therapy (S464L, I491M) $(11,12)$.

Several targeted therapeutic strategies designed to circumvent resistance driven by downstream pathway reactivation are being investigated in ongoing clinical trials that combine an anti-EGFR drug with other targeted therapies such as the HER2 inhibitor trastuzumab or MEK inhibitors $(13,14)$. However, therapeutic strategies to overcome resistance mediated by mutations in EGFR are poorly characterized. A subset of 
mutations that mediate resistance to cetuximab are sensitive to panitumumab $(3,11)$ and as a proof of concept, one patient with a EGFR S492R mutation achieved a partial response to panitumumab (3). However, a subset of EGFR mutations show crossresistance to panitumumab in preclinical models $(11,12)$ and the $G 465 R$ mutation has also been identified in patients progressing to panitumumab (15). Therefore, there remains a significant unmet need for a therapeutic strategy to overcome resistance driven by EGFR mutations.

Sym004 is a 1:1 mixture of two recombinant, human-mouse chimeric mAbs directed against non-overlapping EGFR epitopes (mAb992 and mAb1024). A unique feature of Sym004 is its ability to mediate rapid EGFR internalization and subsequent degradation of the receptor $(16,17)$. Preclinical studies with Sym004 showed superior antitumor activity as compared with other anti-EGFR antibodies as well as activity in models of acquired cetuximab resistance $(16,18)$. Recently, Sym004 has shown promising responses in a phase I clinical trial involving MCRC patients with disease resistant or refractory to cetuximab and/or panitumumab. Interestingly, an EGFR S492R mutation emerged in a tumor biopsy after progression to cetuximab, and this patient achieved a partial response to Sym004 (19). The aim of the present study was to characterize the efficacy of Sym004 to circumvent resistance to cetuximab driven by the emergence of ECD mutations of EGFR. 


\section{Material and Methods}

\section{Cellular models and reagents}

The human colorectal cancer cell lines parental and resistant to cetuximab, parental EGFR WT DiFi and S492R EGFR mutant (DCR7) and parental LIM1215 and LIM1215 G465R cells, were described previously $(3,11)$. Mouse embryonic fibroblast cell line NIH3T3 was incubated with RPMI-1640 medium supplemented with $10 \%$ FBS. All media also contained $2 \mathrm{nmol} / \mathrm{L}$ L-glutamine (Gibco) and antibiotics (100 U/ml penicillin and $100 \mathrm{mg} / \mathrm{ml}$ streptomycin, Gibco). The cells were maintained at $37^{\circ} \mathrm{C}$ in humidified atmosphere with $5 \% \mathrm{CO}_{2}$. The identity of each cell line was tested and authenticated by Cell ID System and by Gene Print 10 System (Promega), through short tandem repeats (STR) at 10 different loci (D5S818, D13S317, D7S820, D16S539, D21S11, vWA, TH01, TPOX, CSF1PO, and amelogenin). Amplicons form multiplex PCRs were separated by capillary electrophoresis (3730 DNA Analyzer, Applied Biosystems) and analyzed using GeneMapperID software form Life Technologies. Resulting cell line STR profiles were cross-compared and matched with the available STR from ATCC, ECACC, and CellBank Australia repositories online database. Cetuximab (Erbitux) and panitumumab (Vectibix) were obtained from the Hospital del Mar clinical pharmacy and Sym004 from Symphogen A/S. We acquired recombinant human EGF protein from Calbiochem.

\section{DNA constructs and mutagenesis}

The EGFR DNA constructs (S492R, G465R, R451C, and K467T) created by sitedirected mutagenesis were described previously. The presence of mutations was confirmed by DNA direct sequencing $(3,11)$.

\section{Flow Cytometry}

To determine cetuximab and Sym004 binding to cells that stably express the EGFR mutants, the cells were trypsinized and washed with PBS twice. Then, the samples 
were incubated with Fc blocking solution (MiltenyiBiotec) for 15 minutes on ice to block nonspecific Fc binding of immunoglobulins. The samples were then washed twice with PBS and incubated with the monoclonal antibodies for EGFR binding, cetuximab or Sym004, during 30 minutes at $100 \mathrm{ng} / \mathrm{ml}$ on ice. To visualize the primary antibody a goat anti-human IgGy phycoerythrin-conjugated (Invitrogen) was used as a secondary antibody. EGFR binding was analyzed using the FACScan flow Cytometer (BD Bioscience) in the Flow Cytometry Core Facility of Barcelona Biomedical Research Park (PRBB).

\section{Protein detection}

We subjected total cell lysates to Western blot analysis as previously reported (3). The following antibodies were used for protein detection: phospho EGFR (Y1068), phospho ERK1/2 (Thr202/Tyr204), phospho AKT (Ser473), EGFR, ERK1/2 and AKT. All the antibodies were purchased from Cell Signaling Technology. Antibody to a-tubulin was obtained from Sigma.

\section{Cell viability determination by crystal violet staining assay}

To analyze the growth inhibitory effect of cetuximab and Sym004 in long-term experiments, we plated $6 \times 10^{3}$ cells per well in a 6-well plate with culture medium containing $10 \%$ FBS. After 24 h, cetuximab, panitumumab or Sym004 were added at 0.1 or $10 \mu \mathrm{g} / \mathrm{ml}$, and incubated during 10-15 days. The medium was changed 3 times a week. Cell viability was determined by crystal violet staining at $0.1 \%$ for $1 \mathrm{~h}$. Quantification was evaluated using ImageJ software. The results were plotted as percentage of control.

\section{Molecular Simulations}


The coordinates of the cetuximab:EGFR complex were downloaded from the RCSB protein data bank (pdb ID code 1YY9). The positions of EGFR mutants on domain III were visualized with PyMOL software.

\section{Samples from patients}

Formalin-fixed and paraffin-embedded tumor biopsy samples from metastatic refractory colorectal cancer patients included in a phase I clinical trial with Sym004 (19) were sent to Hospital del Mar Molecular Laboratory. Biopsies were performed before the first infusion of Sym004. DNA was extracted and mutations in exon 12 of EGFR, exon 3,4,5 of KRAS, exons $3,4,5$ of NRAS, exon 15 of BRAF and exons 9, 20 of PIK3CA were analyzed by pyrosequencing using, a next generation sequencing (NGS) 454 GS Junior platform (Roche Applied Science) and by MiSeq (Illumina) as previously reported (11).

\section{Subcutaneous tumorigenesis}

Five-week-old male BALB/c nude mice were purchase from Charles River Laboratories and hosted under standard conditions in the pathogen-free animal facility at the Barcelona Biomedical Research Park (PRBB). Animal treatments were done according to institution-approved protocols. Mice were treated humanely and with regard for alleviation of suffering. Cells were suspended in sterile PBS with $50 \%$ Matrigel (BD Biosciences) and $5 \times 10^{6}$ cells were injected subcutaneously into the flank of mice. Tumor volume was determined from caliper measurements of tumor length $(L)$ and width (W) according to the formula $L \times W^{2} / 2$ twice a week. Tumors were allowed to grow until the volume reached approximately 200 to $300 \mathrm{~mm}^{3}$. To analyze the S492R EGFR mutation mice were randomized to 6 groups with 5 mice in each group, 3 groups injected with DiFi cells and 3 with DCR7 cells. Treatment groups consisted of DiFi control (IgG Isotype Control), DCR7 control (IgG Isotype Control), DiFi + CTX (40 $\mathrm{mg} / \mathrm{kg}$ of cetuximab), DCR7 + CTX (40 mg/kg), DiFi + Sym004 (40 mg/kg), and DCR7 
+ Sym004 (40 mg/kg). To analyze the G465R EGFR mutation mice were randomized to 8 groups with 5 mice in each group, 4 groups injected with DiFi cells and 4 with DCR7 cells. Treatment groups consisted of LIM1215 control (IgG Isotype Control), LIM1215 G465R control (IgG Isotype Control), LIM1215 + CTX (40 mg/kg of cetuximab), LIM1215 G465R + CTX (40 mg/kg), LIM1215 + PNM (40 mg/kg of panitumumab), LIM1215 G465R + PNM (40 mg/kg), Lim1215 + Sym004 (40 mg/kg), and LIM1215 G465R + Sym004 (40 mg/kg). Mice were treated intraperitoneally twice a week.

\section{Statistical analysis of data}

Statistical analyses were carried out using one-way ANOVA tests followed by post hoc Tukey adjustment. For the MTS assay two-way ANOVA tests followed by post hoc Bonferroni adjustment was done. Prism 5.0 software (GraphPad) was used for the statistical analyses. Significance was set at $p<0.05$. Data shown are mean \pm SD. 


\section{Results}

Contrary to cetuximab and panitumumab, Sym004 effectively binds to and prevents activation of all the EGFR mutants

To assess the ability of Sym004 to interact with EGFR harboring ectodomain mutations identified in patients (S492R, R451C, K467T, and G465R), wild-type and mutant EGFR were ectopically expressed in NIH 3 T3 cells that lack endogenous EGFR. Flow cytometry was used to establish the extent of cetuximab, panitumumab and Sym004 binding to cells expressing the mutants as compared to wild-type EGFR. These experiments clearly showed that EGFR mutations were not permissive for binding to cetuximab, providing functional evidence of their role in driving acquired resistance to this drug. On the other hand, while only a subset of EGFR mutations was permissive for binding to panitumumab, Sym004 was effectively binding to all mutants including the one harboring the G465R mutation (Table 1, Figure 1A, Supplementary Figure S1). Of note, the effect of EGFR R451C on cetuximab, panitumumab, and Sym004 binding was moderate compared to the other mutants. Analysis of the published cetuximab:EGFR crystal structure confirmed that all EGFR mutations tested are located in the surface recognized by cetuximab, with the exception of the R451C mutation (Figure 1B).

To further characterize the functional properties of EGFR mutations, we performed competitive studies to determine the capacity of the three drugs to revert the activation of the receptor, via phosphorylation of the tyrosine 1068 , in the presence of its natural ligand EGF. As expected, cetuximab abrogated ligand-mediated activation of the wildtype receptor, while had no or very limited impact in cells carrying individual EGFR mutants. Notably, cetuximab was still inhibiting phosphorylation of the EGFR R451C mutant receptor. Panitumumab was preventing the phosphorylation of all EGFR mutants except for the EGFR p.G465R. On the contrary, Sym004 effectively prevented phosphorylation of all EGFR mutants including the EGFR p.G465R one (Table 1, 
Figure 1C). To better characterize the effects of the G465R mutation, we assessed the capacity of the different antibodies to block the phosphorylation of EGFR p.G465R in the presence of increasing concentrations of the high-affinity natural ligand EGF. While Sym004 showed a potent blockade of EGFR phosphorylation even in the presence of high concentrations of EGF, cetuximab was not able to prevent ligand-induced phosphorylation of EGFR at any given concentration of EGF (Figure 1D).

Sym004 effectively inhibits proliferation and EGFR downstream signaling in cetuximab-resistant derivatives harboring the S492R and G465R EGFR mutations Among the EGFR mutations identified in patients, the S492R and the G465R EGFR mutations have also spontaneously emerged upon continuous exposure to cetuximab in CRC cell lines, offering an interesting preclinical model to assess the cell viability and molecular effects of Sym004 in vitro.

We first characterized the effects of Sym004 on the DiFi cetuximab-resistant cells (DCR7) harboring the EGFR S492R mutation. Long-term cell viability assay showed that both panitumumab and Sym004 were effectively inhibiting the proliferation of the parental DiFi as well as DCR7 cells, as compared to cetuximab that was only effective in DiFi parental cells (Figure 2A and Supplementary Figure S2A). Sensitivity to Sym004 and panitumumab correlated with an effective abrogation of the phosphorylation of the downstream effectors AKT and ERK, being the effects on AKT more evident with Sym004 (Figure 2B). Sym004 also effectively inhibited proliferation of the LIM1215 G465R cell line, as observed with a significant reduction on the longterm cell cloning capacity, whereas effects of panitumumab and cetuximab were not significant (Figure 2C and Supplementary Figure S2B). Biochemical analysis revealed that sensitivity to Sym004 was consistent with a detectable attenuation of signaling to the downstream effectors ERK and AKT in LIM1215 G465R mutant cells (Figure 2D). On the contrary, panitumumab did not significantly impact cell viability of G465R 
mutant cells (Figure 2C and supplementary Figure S2B) and did not inhibit phosphorylation of the downstream effectors ERK and AKT (Figure 2D).

\section{Sym004 causes profound and sustained regression in S492R mutant EGFR and}

\section{delays tumor growth in G465R mutant EGFR in vivo}

Parental EGFR wild-type DiFi, S492R EGFR mutant DCR7, parental EGFR wild-type LIM1215, and G465R EGFR mutant LIM1215 cell lines were injected in the flanks of immunodeficient mice ( $\mathrm{n}=5$ in each group) to establish tumor xenografts. To analyze the in vivo antitumor efficacy of anti-EGFR therapy, we treated the mice with cetuximab, panitumumab, or Sym004 twice a week. We observed that large tumors were generated in the control groups within the first weeks independently from the injected cell lines (Figures $3 A-B$ and $4 A-B$ ). Tumor growth was not detected for cetuximab or Sym004 treated mice with DiFi cells xenografts (Figure 3A). However, in cetuximab treated S492R EGFR mutant DCR7 xenografts, an increase in tumor growth rate was observed after approximately 40 days (Figure 3B). Strikingly, in the group of Sym004 treated S492R EGFR mutant DCR7 cells injected-mice, no tumor growth was observed along the whole experiment (Figure 3B), suggesting that Sym004 is able to induce a profound and sustained tumor regression. When injecting the mice with parental LIM1215 cells and treated with cetuximab, panitumumab, or Sym004, we observed significantly less tumor growth than in the control condition (Figure 4A). In the groups of cetuximab and panitumumab treated G465R EGFR mutant LIM1215 injected-mice, the tumors proliferated at a comparable rate to control-treated xenografts. Importantly, Sym004 significantly delayed tumor growth in G465R EGFR mutant LIM1215 xenografts (Figure 4B). 


\section{Proof-of-principle clinical activity of Sym004 in a MCRC patients with acquired resistance to cetuximab mediated by an EGFR G465R mutation}

To gain further insight into the clinical activity of Sym004, we obtained tissue samples from 13 refractory MCRC patients treated with Sym004 in a phase I clinical trial (19). The analyzed tumor samples corresponded to biopsies obtained after progression to cetuximab/panitumumab, and prior to Sym004 treatment initiation. Genomic DNA was extracted and exploited to ascertain the mutational profiling of genes known to be involved in the EGFR signalling axis. Acquired resistance to cetuximab was associated with the emergence of mutations, which occurred mainly in KRAS, NRAS and EGFR genes (Table 2). An EGFR G465R mutation (22\% of mutant alleles) was detected in a biopsy from a patient which had been previously treated with cetuximab. As a proof of principle, subsequent treatment of this patient with Sym004 at yielded a stabilization of the disease lasting 15 weeks. Although this is a promising observation, further evidence of the clinical relevance of Sym004 in patients with resistance to cetuximab/panitumumab harbouring a G465 EGFR mutation is needed. In this context, a clinical trial to assess the efficacy of Sym004 in patients with EGFR ECD mutations is ongoing. 


\section{Discussion}

Targeting EGFR is an approved clinical strategy for the treatment of patients with KRAS and NRAS wild-type MCRC. Unfortunately, responses are transient, and acquired resistance inevitably emerges which limits the clinical efficacy of these drugs(1, 2). Ascertainment of the molecular landscape of resistant tumors has shown that colorectal cancer cells evade EGFR blockade by two main strategies, including downstream pathway reactivation (mainly KRAS and NRAS mutations) and EGFR ECD mutations that occurs in $15-20 \%$ of patients $(3-5,9,11,12)$. No specific treatments for patients with acquired resistance to current anti-EGFR mAb have yet been approved. Here, we describe Sym004 as a valid strategy to effectively bypass resistance driven by mutations in EGFR in patients previously treated with anti-EGFR $\mathrm{mAb}$.

So far, four mutations in the ECD of EGFR have been identified to drive resistance to approved anti-EGFR mAb in patients: S492R, R451C, K467T after cetuximab and G465R after panitumumab failure. Two more mutations have been reported in cell lines only (S464L, 1491M). These mutations occur in key aminoacids located in the binding epitope of the mAb that impair the affinity of the drug to the receptor $(3,11,15)$. Interestingly, resistance is caused by the failure of the antibody to bind to its receptor, but tumors still retain their dependence on EGFR, which suggests that therapeutic strategies administered after the development of resistance to cetuximab or panitumumab should still include an EGFR inhibitor. Sym004 is a mixture of two synergistic anti-EGFR monoclonal (992 and 1024) antibodies directed against distinct non-overlapping epitopes in EGFR extracellular domain III that has shown promising clinical activity in phase 1/2 (19). Specific for Sym004, mAb 992 has been shown to bind contact residues K467, I491, and S492, whereas mAb 1024 has been shown to bind to S484 and G485 (17). The larger binding region of the sum of the two antibodies in Sym004 confers an advantage to Sym004 in reverting resistance driven by point mutations in EGFR. Of note, R451C is the only EGFR mutation not located in the 
cetuximab, panitumumab or Sym004 binding site. However, the mutation may lead to critical structural changes that impair antibody-receptor binding. It is also worthnoting that R451C has always been concurrently detected with mutations in KRAS/NRAS within the same tumor sample (11). Furthermore, compared to anti-EGFR mAbs currently approved for clinical use, Sym004 does not only block ligand-binding receptor activation and downstream receptor signaling, but also induces rapid EGFR internalization and degradation $(16,20)$.

Of special interest is the G465R mutation that has been shown to emerge in cetuximab-resistant cell lines as well as in patients with disease progressing to panitumumab. This mutation is effectively targeted by Sym004 in our preclinical functional studies. Moreover, as a proof-of-concept, a patient with cetuximab resistance harboring the G465R mutation responded to treatment with Sym004. Similarly, the S492R mutation has also previously been reported in a patient progressing to cetuximab therapy that responded to subsequent treatment with Sym004 (19). Of note, the percentage of EGFR mutations found in the present study was lower than previously reported, in part due to different sensitivity of the detection techniques in the studies as well as the recent advantage of circulating tumor DNA in capturing the heterogeneity of solid tumors as compared to single biopsies of one tumor lesion $(8,21)$.

Importantly, Sym004 could also offer an attractive treatment option in anti-EGFR naïve patients, alone or in combination with chemotherapy, through targeting both wild-type EGFR population and delaying the emergence of mutant EGFR tumor cells. However, it remains to be determined in the clinics whether a delay of disease progression and increase in survival will be achieved through treatment with Sym004 after cetuximab progression or earlier in MCRC patients.

To sum up, $15-20 \%$ of MCRC evade EGFR blockade through EGFR extracellular mutations. Our data suggest that Sym004 is an effective strategy to bypass resistance driven by mutations in EGFR. The identification of mutations of EGFR in patients 
resistant to EGFR blockade is paramount to design additional lines of therapy that include Sym004.

\section{Acknowledgements}

We thank Fundació Cellex (Barcelona) for a generous donation to the Hospital del Mar Medical Oncology Service. 


\section{References}

1. Van Cutsem E, Kohne CH, Hitre E, Zaluski J, Chang Chien CR, Makhson A, et al. Cetuximab and chemotherapy as initial treatment for metastatic colorectal cancer. $\mathrm{N}$ Engl J Med. 2009;360:1408-17.

2. Douillard JY, Oliner KS, Siena S, Tabernero J, Burkes R, et al. PanitumumabFOLFOX4 treatment and RAS mutations in colorectal cancer. $\mathrm{N}$ Engl $\mathrm{J}$ Med. 2013;369:1023-34.

3. Montagut C, Dalmases A, Bellosillo B, Crespo M, Pairet S, Iglesias M, et al. Identification of a mutation in the extracellular domain of the Epidermal Growth Factor Receptor conferring cetuximab resistance in colorectal cancer. Nat Med. 2012;18:2213.

4. Misale S, Yaeger R, Hobor S, Scala E, Janakiraman M, Liska D, et al. Emergence of KRAS mutations and acquired resistance to anti-EGFR therapy in colorectal cancer. Nature. 2012;486:532-6.

5. Diaz LA, Jr., Williams RT, Wu J, Kinde I, Hecht JR, Berlin J, et al. The molecular evolution of acquired resistance to targeted EGFR blockade in colorectal cancers. Nature. 2012;486:537-40.

6. Bardelli A, Corso S, Bertotti A, et al. Amplification of the MET receptor drives resistance to anti-EGFR therapies in colorectal cancer. Cancer Discov. 2013;3:658673.

7. Yonesaka K, Zejnullahu K, Okamoto I, et al. Activation of ERBB2 signaling causes resistance to the EGFR-directed therapeutic antibody cetuximab. Sci Transl Med. 2011;3:99ra86.

8. Siravegna G, Mussolin B, Buscarino M, Corti G, Cassingena A, Crisafulli G, et al. Clonal evolution and resistance to EGFR blockade in the blood of colorectal cancer patients. Nat Med. 2015;21:795-801. 
9. Morelli MP, Overman MJ, Dasari A, Kazmi SM, Mazard T, Vilar E, et al. Characterizing the patterns of clonal selection in circulating tumor DNA from patients with colorectal cancer refractory to anti-EGFR treatment. Ann Oncol. 2015;26:731-6.

10. Newhall K, Price T, Peeters M, Kim TW, Li J, Cascinu S, et al. Frequency of S492R Mutations in the Epidermal Growth Factor Receptor: Analysis of Plasma DNA from Metastatic Colorectal Cancer Patients treated with Panitumumab or Cetuximab Monotherapy. Annals of Oncology. 2014;25:ii109.

11. Arena S, Bellosillo B, Siravegna G, Martinez A, Canadas I, Lazzari L, et al. Emergence of Multiple EGFR Extracellular Mutations during Cetuximab Treatment in Colorectal Cancer. Clin Cancer Res. 2015;21:2157-66.

12. Braig F, Marz M, Schieferdecker A, Schulte A, Voigt M, Stein A, et al. Epidermal growth factor receptor mutation mediates cross-resistance to panitumumab and cetuximab in gastrointestinal cancer. Oncotarget. 2015;6:12035-47.

13. Siena S, Sartore-Bianchi A, Lonardi S, Trusolino L, Martino C, Bencardino K, et al. Trastuzumab and lapatinib in HER2-amplified metastatic colorectal cancer patients (mCRC): The HERACLES trial. ASCO Meeting Abstracts. 2015;33:3508.

14. Misale S, Arena S, Lamba S, Siravegna G, Lallo A, Hobor S, et al. Blockade of EGFR and MEK intercepts heterogeneous mechanisms of acquired resistance to antiEGFR therapies in colorectal cancer. Sci Transl Med. 2014;6:224ra26.

15. Voigt M, Braig F, Gothel M, Schulte A, Lamszus K, Bokemeyer C, et al. Functional dissection of the epidermal growth factor receptor epitopes targeted by panitumumab and cetuximab. Neoplasia. 2012;14:1023-31.

16. Pedersen MW, Jacobsen HJ, Koefoed K, Hey A, Pyke C, Haurum JS, et al. Sym004: a novel synergistic anti-epidermal growth factor receptor antibody mixture with superior anticancer efficacy. Cancer Res. 2010;70:588-97.

17. Koefoed K, Steinaa L, Soderberg JN, Kjaer I, Jacobsen HJ, Meijer PJ, et al. Rational identification of an optimal antibody mixture for targeting the epidermal growth factor receptor. MAbs. 2011;3:584-95. 
18. lida M, Brand TM, Starr MM, Li C, Huppert EJ, Luthar N, et al. Sym004, a novel EGFR antibody mixture, can overcome acquired resistance to cetuximab. Neoplasia. 2013;15:1196-206.

19. Dienstmann R, Patnaik A, Garcia-Carbonero R, Cervantes A, Benavent M, Rosello S, et al. Safety and Activity of the First-in-Class Sym004 Anti-EGFR Antibody Mixture in Patients with Refractory Colorectal Cancer. Cancer Discov. 2015;5:598-609.

20. Skartved NJ, Jacobsen HJ, Pedersen MW, Jensen PF, Sen JW, Jorgensen TK, et al. Preclinical pharmacokinetics and safety of Sym004: a synergistic antibody mixture directed against epidermal growth factor receptor. Clin Cancer Res. 2011;17:5962-72.

21. Bettegowda C, Sausen M, Leary RJ, Kinde I, Wang $\mathrm{Y}$, Agrawal N, et al. Detection of circulating tumor DNA in early- and late-stage human malignancies. Sci TransI Med. 2014;6:224ra24. 
Table 1. EGFR ECD mutations identified in patients with colorectal cancer and response to anti-EGFR drugs cetuximab (CTX), panitumumab (PNM) and Sym004, including summary of the functional, cell viability, biochemical studies and clinical results.

\begin{tabular}{|c|c|c|c|c|c|c|c|c|c|c|c|c|c|}
\hline \multirow[t]{2}{*}{$\begin{array}{l}\text { EGFR } \\
\text { mutation }\end{array}$} & \multicolumn{3}{|c|}{ Drug - receptor binding } & \multicolumn{3}{|c|}{$\begin{array}{l}\text { Inhibition of EGFR } \\
\text { phosphorylation }\end{array}$} & \multicolumn{3}{|c|}{ Drug sensitivity } & \multicolumn{3}{|c|}{$\begin{array}{l}\text { EGFR downstream signaling } \\
\text { inhibition }\end{array}$} & \multirow{2}{*}{$\begin{array}{r}\text { Pat } \\
\text { Res } \\
\text { PNM }\end{array}$} \\
\hline & CTX & PNM & Sym004 & CTX & PNM & Sym004 & CTX & PNM & Sym004 & CTX & PNM & Sym004 & \\
\hline S492R & no & yes & yes & no & yes & yes & $\mathrm{R}$ & $S$ & $S$ & no & yes & yes & yes \\
\hline R451C & moderate & moderate & moderate & yes & yes & yes & * & * & * & * & * & $\star$ & - \\
\hline K467T & no & yes & yes & no & yes & yes & $\star$ & $\star$ & $\star$ & * & * & * & - \\
\hline G465R & no & no & yes & no & no & yes & R & $\mathrm{R}$ & $S$ & no & no & yes & no \\
\hline
\end{tabular}


Table 2. KRAS, NRAS, and EGFR ECD point mutations in tumor biopsies after progression to cetuximab and before initiation of treatment with Sym004 in patients with metastatic colorectal cancer.

\begin{tabular}{|c|c|c|c|c|c|c|c|c|c|c|}
\hline pt \# & & KRAS & & & NRAS & & & & $G F R$ & \\
\hline & EXON2 & EXON3 & EXON4 & EXON2 & EXON3 & EXON4 & R451C & S492R & G465R & K467T \\
\hline 1 & & & & & & & & & & \\
\hline 2 & & & & & & & & & & \\
\hline 3 & & & & & & & & & & \\
\hline 4 & & & & & & & & & & \\
\hline 5 & & & & & & & & & & \\
\hline 6 & & & & & & & & & & \\
\hline 7 & & & & & & & & & & \\
\hline 8 & & & & & & & & & & \\
\hline 9 & & & & & & & & & & \\
\hline 10 & & & & & & & & & & \\
\hline 11 & & & & & & & & & & \\
\hline 12 & & & & & & & & & & \\
\hline 13 & & & & & & & & & & \\
\hline & & & $<20 \%$ & & & & & & & \\
\hline & & & $20-50 \%$ & & & & & & & \\
\hline & & & $>50 \%$ & & & & & & & \\
\hline
\end{tabular}




\section{Figure Legends}

Figure 1. Functional analysis of cetuximab (CTX), panitumumab (PNM), and Sym004 in NIH-3T3 cells expressing the individual EGFR ECD mutations (A) Contrary to cetuximab and panitumumab, Sym004 effectively bound to all EGFR mutants. NIH 3T3 cells expressing wild-type (WT) EGFR or the indicated EGFR mutations were incubated with CTX, PNM, or Sym004 $(10 \mu \mathrm{g} / \mathrm{ml})$, and antibody binding was analyzed by flow cytometry using a secondary antibody to human IgG conjugated with phycoerythrin (PE). NIH 3T3 cells expressing the empty vector were used as a negative control (empty). Cell binding was normalized to the binding of EGFR WT cells. (B) Molecular visualization of cetuximab binding to EGFR domain III. EGFR mutations are highlighted and shown to overlap with the cetuximab binding site, except for the distal p.R451C mutation that is present on the lower part of domain III (C) Contrary to cetuximab and panitumumab, Sym004 effectively prevented phosphorylation of all EGFR mutations. NIH 3 T3 cells expressing WT EGFR or the indicated EGFR mutations were cultured in the presence of CTX, PNM, or Sym004 $(10 \mu \mathrm{g} / \mathrm{ml})$ for 2 hours and stimulated with EGF (5 ng/ml) for 15 minutes. Immunoblots were performed using antibodies to the indicated proteins. (D) Effects of cetuximab, panitumumab and Sym004 on EGFR G465R phosphorylation in the presence of increasing concentrations of EGF.NIH 3T3 cells expressing the EGFR p.G465R mutation were cultured in the presence of CTX, PNM, or Sym004 $(10 \mu \mathrm{g} / \mathrm{ml})$ for 2 hours and stimulated with $\operatorname{EGF}(0,2.5,5,10,50$, and $100 \mathrm{ng} / \mathrm{ml})$ for 15 minutes. Immunoblots were performed using antibodies to the indicated proteins.

Figure 2. Effects of cetuximab (CTX), panitumumab (PNM), and Sym004 on proliferation and EGFR downstream signaling in cetuximab-resistant cells harboring the S492R mutation and the G465R mutation, generated from sensitive parental cells. (A) Clonogenic capacity analyzed by staining the cells with crystal 
violet after treating the cells with 0.1 or $10 \mu \mathrm{g} / \mathrm{ml}$ of CTX, PNM, or Sym004 during 1215 days (B) Biochemical analysis revealing an effective suppression of AKT and ERK following PNM and Sym004 therapy. Cell were cultured in the presence of CTX, PNM, or Sym004 (10 $\mu \mathrm{g} / \mathrm{ml})$ for 2 hours. Immunoblots were performed using antibodies to the indicated proteins. $\left(^{*}\right) p<0.05$. (C) Clonogenic capacity analyzed by staining the cells with crystal violet after treating the cells with 0.1 or $10 \mu \mathrm{g} / \mathrm{ml}$ of CTX, PNM, or Sym004 during 12-15 days. (D) Cells were cultured in the presence of CTX, PNM, or Sym004 $(10 \mu \mathrm{g} / \mathrm{ml})$ for 2 hours, followed by 15 minutes with EGF $(5 \mathrm{ng} / \mathrm{ml})$. Immunoblots were performed using antibodies to the indicated proteins. $\left(^{*}\right) p<0.05$.

Figure 3. Effects of cetuximab (CTX) and Sym004 on colorectal cancer tumor xenografts harboring the S492R mutation. Mice were injected subcutaneously in the dorsal flank with parental EGFR WT DiFi or S492R EGFR mutant DCR7 cell lines as described in the Materials and Methods. After 2 weeks (average tumor size 200-300 $\mathrm{mm}^{3}$ ) mice were treated i.p. twice a week with: IgG Isotype Control, CTX (40 mg/kg), or Sym004 (40 mg/kg). Each group consisted of 5 mice. Tumor volumes were normalized individually to their volumes at the first day of treatment. CTX and Sym004 significantly reduced tumor growth in DiFi injected-mice $(p<0.0001)$. In S492R EGFR mutant DiFi injected-mice Sym004 significantly suppressed tumor growth $(p<0.0001)$.

Figure 4. Effects of cetuximab (CTX), panitumumab (PNM), and Sym004 on colorectal cancer tumor xenografts harboring the G465R mutation. Mice were injected subcutaneously in the dorsal flank with parental EGFR WT LIM1215 or G465R EGFR mutant LIM1215 cell lines. After 2 weeks (average tumor size 200-300 mm³) mice were treated i.p. twice a week with: IgG Isotype Control, CTX (40 mg/kg), PNM (40 mg/kg), or Sym004 (40 mg/kg). Each group consisted of 5 mice. Tumor volumes were normalized individually to their volume on first day of treatment. CTX, PNM, and Sym004 significantly reduced tumor growth in LIM1215 injected-mice $(p<0.05)$. In 
G465R EGFR mutant LIM1215 injected-mice, Sym004 significantly delayed tumor growth $(p<0.05)$. 
(A)

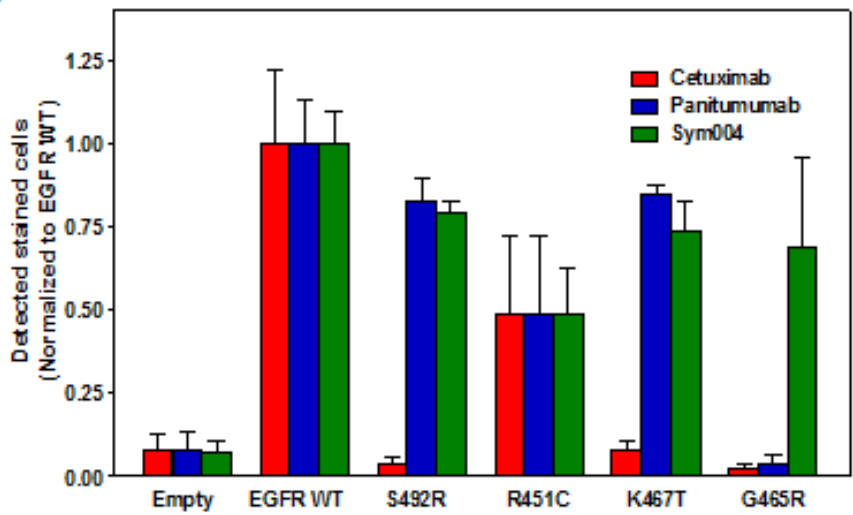

(C)

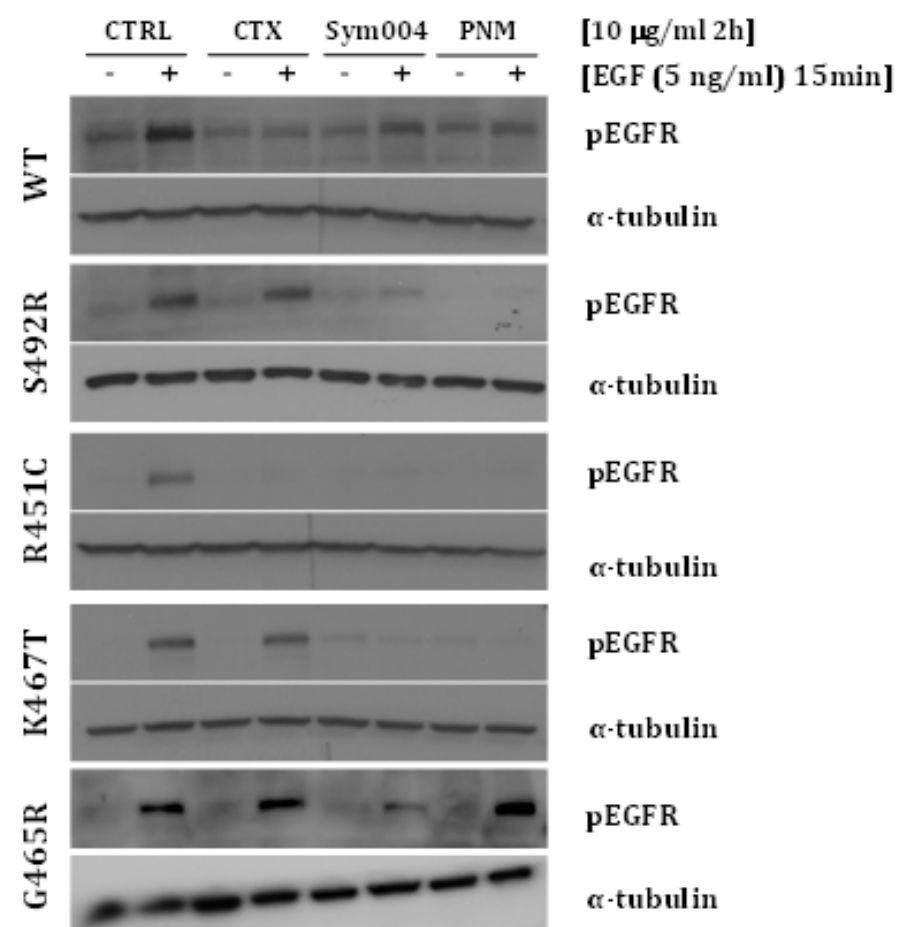

(B)

\section{Sánchez-Martín et al. Figure 1}

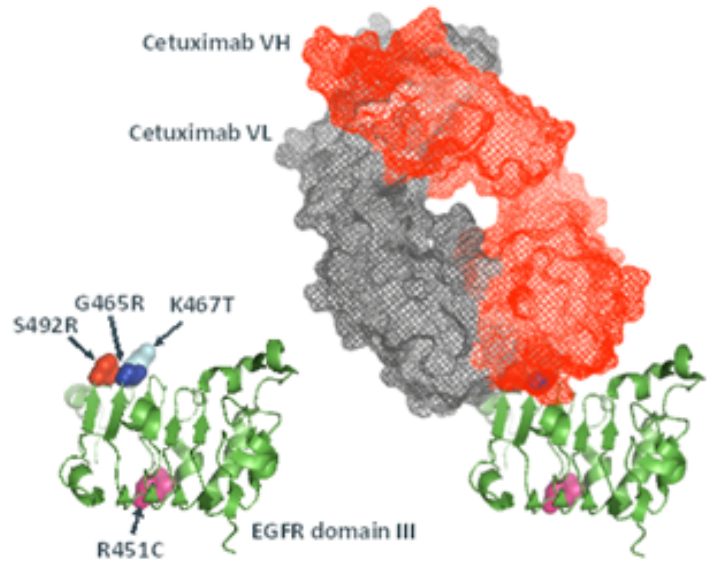

(D)

\section{EGFR G465R}
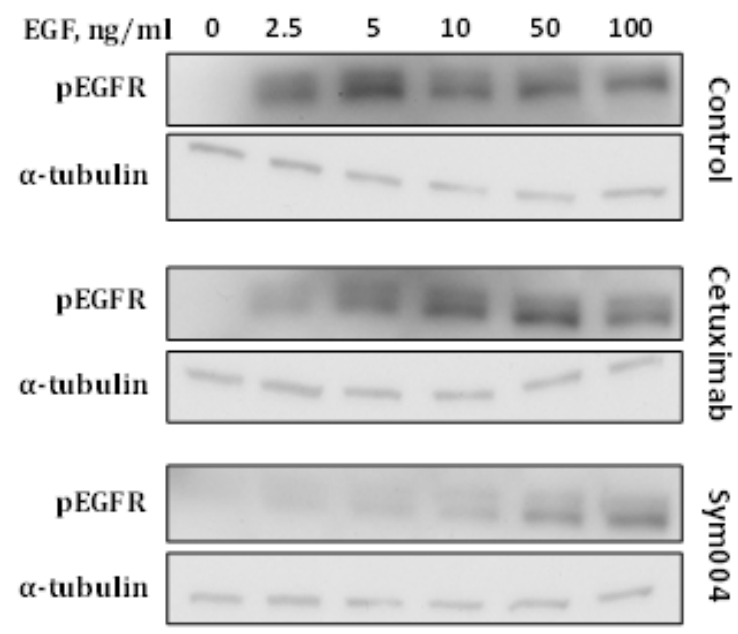
(A)

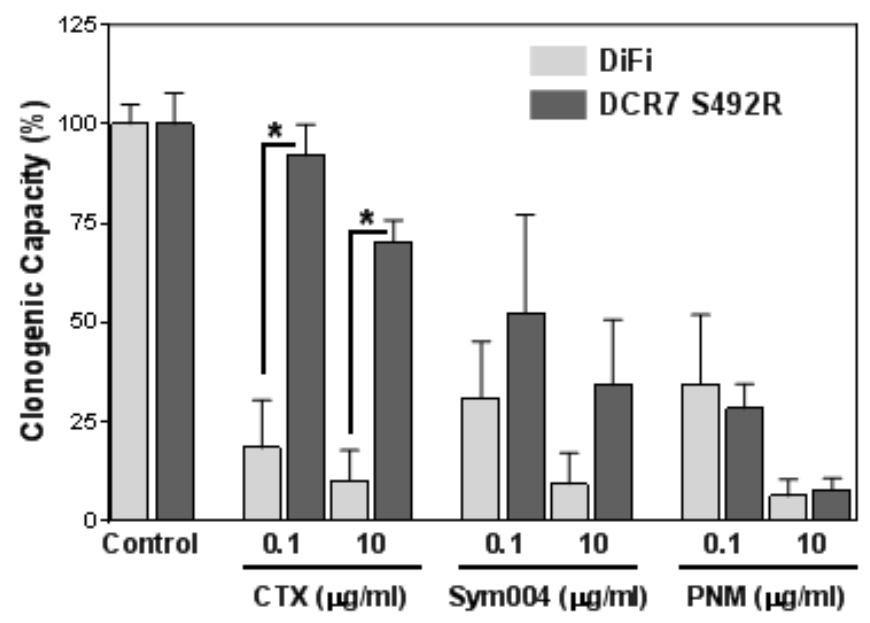

(C)

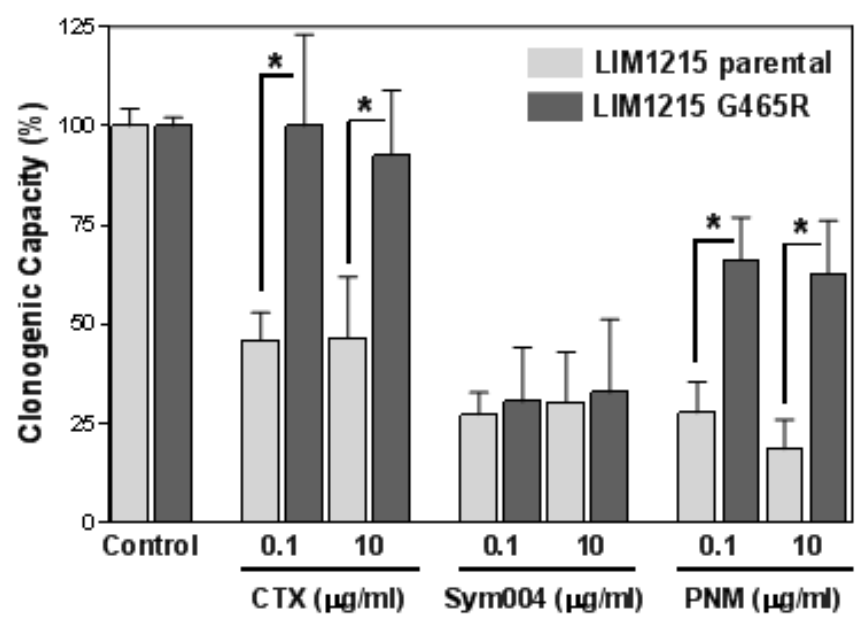

(B)

Sánchez-Martín et al. Figure 2

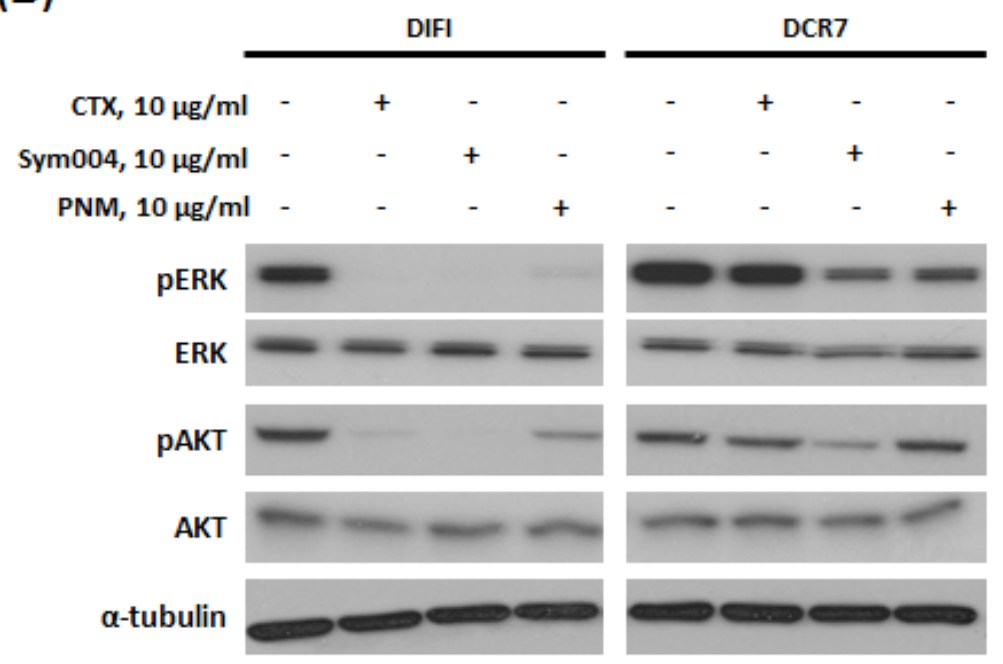

(D)

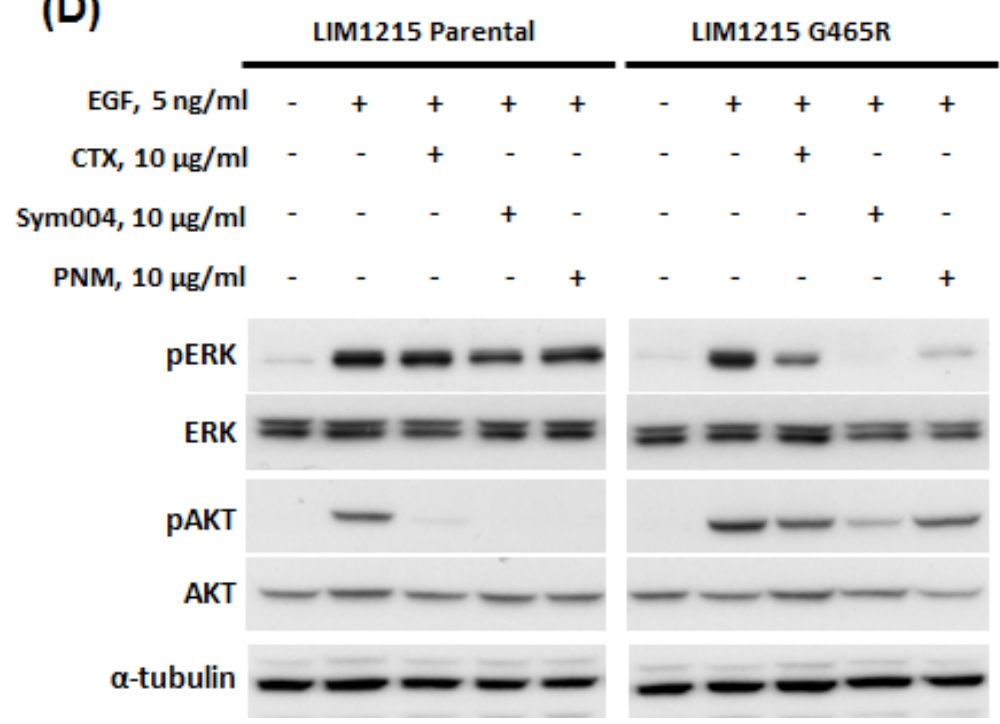


(A)

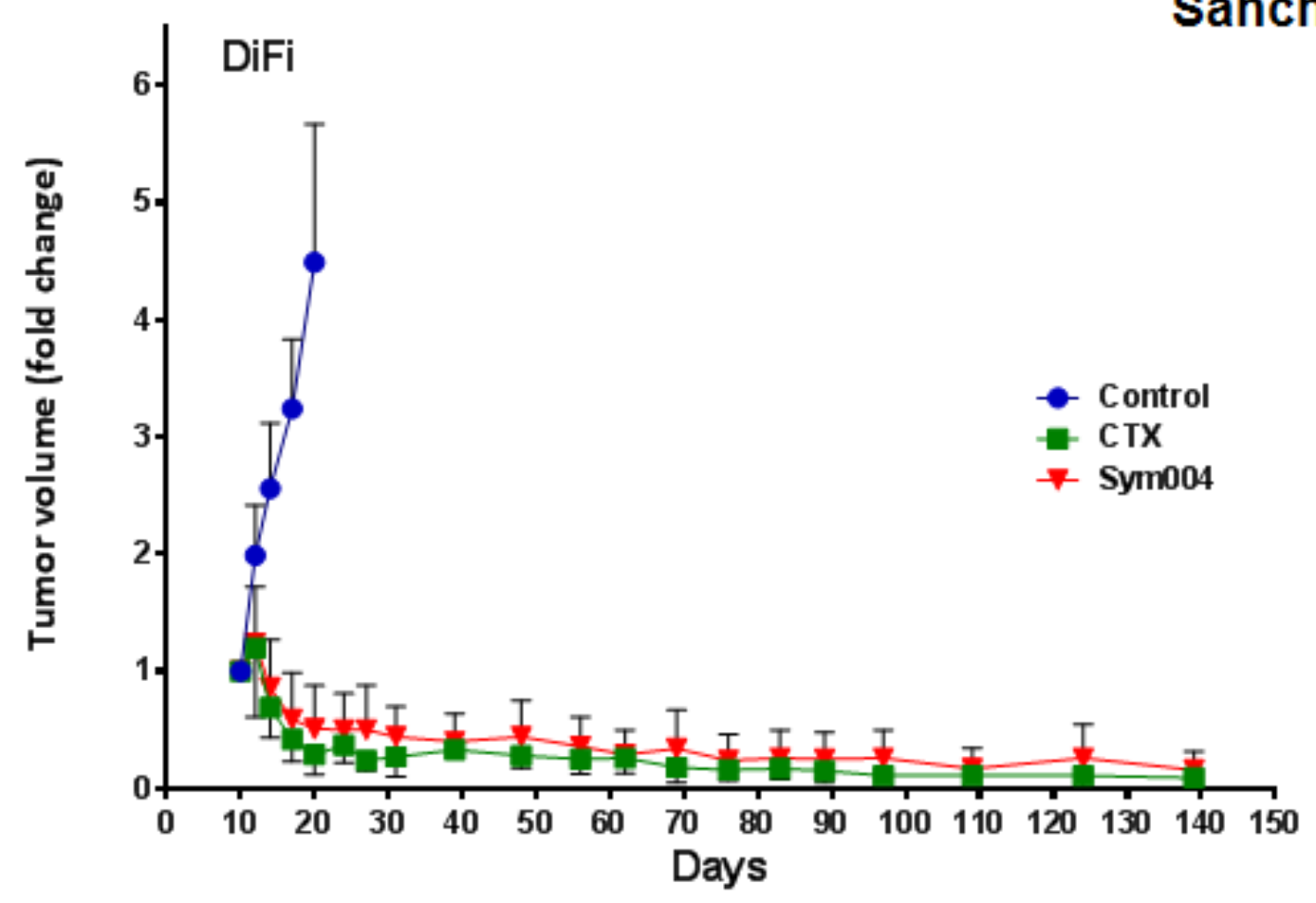

(B)

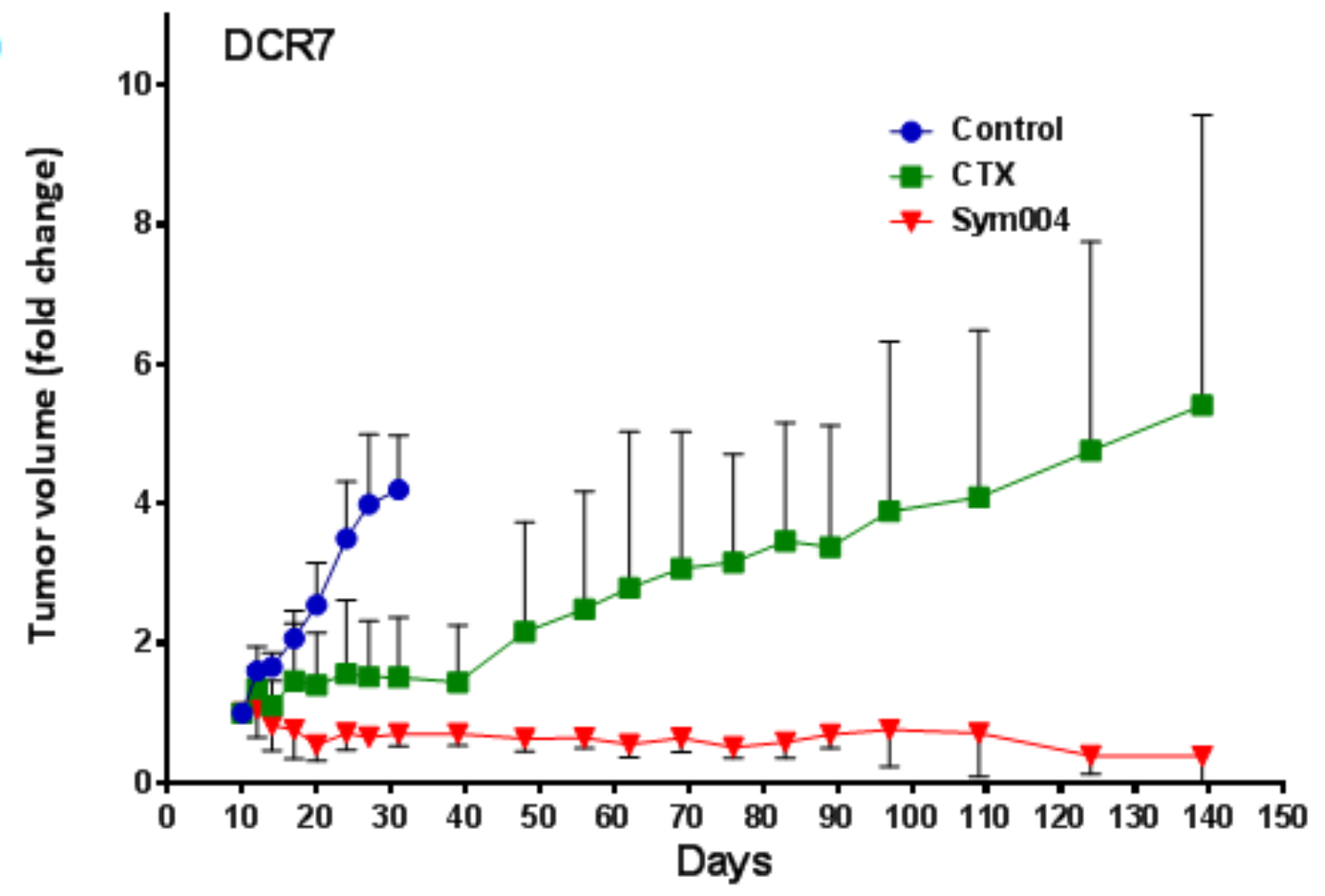


(A)

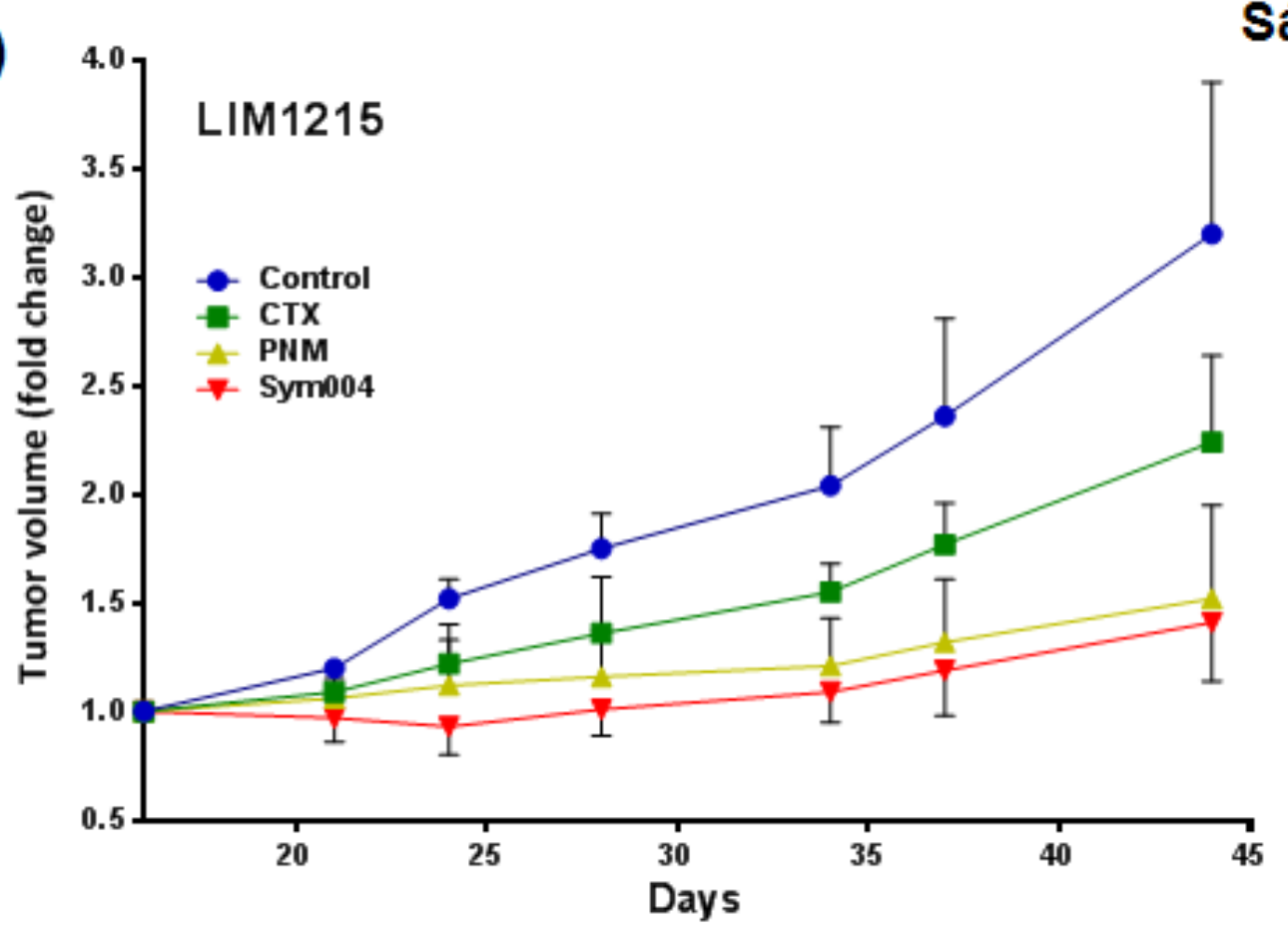

Sánchez-Martín et al. Figure 4

(B)

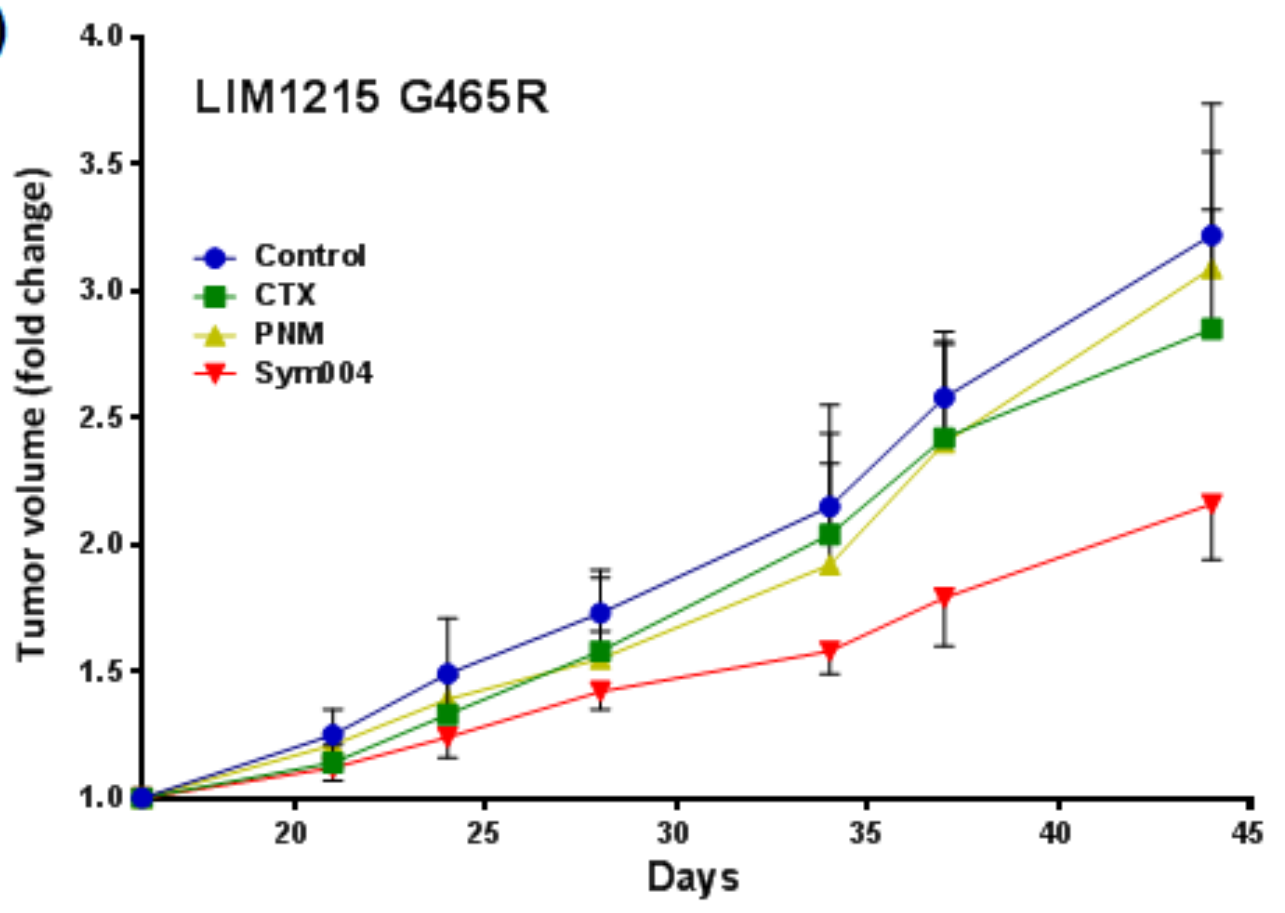

